\title{
Fluxonium: single Cooper pair circuit free of charge offsets
}

\author{
Vladimir E. Manucharyan, ${ }^{1}$ Jens Koch, ${ }^{1}$ Leonid I. Glazman, ${ }^{1}$ Michel H. Devoret ${ }^{1}$ \\ ${ }^{1}$ Departments of Physics and Applied Physics, Yale University, New Haven, Connecticut 06520, USA
}

\begin{abstract}
The promise of single Cooper pair quantum circuits based on tunnel junctions for metrology and quantum information applications is severely limited by the influence of "offset" charges - random, slowly drifting microscopic charges inherent to many solid-state systems. By shunting a small junction with the Josephson kinetic inductance of a series array of large capacitance tunnel junctions, thereby ensuring that all superconducting islands are connected to the circuit by at least one large junction, we have realized a new superconducting artificial atom which is totally insensitive to offset charges. Yet, its energy levels manifest the anharmonic structure associated with single Cooper pair effects, a useful component for solid state quantum computation.
\end{abstract}

Electric charge can be manipulated at the level of a single charge quantum (1) in two types of superconducting circuits with different topologies. The minimal example of the first type of circuit is the Cooper pair box, which consists of an isolated superconducting electrode ("island") connected to a superconducting reservoir on one side by a small tunnel junction, and on the other side by a gate capacitance in series with a voltage source. The dynamics of the island is described by two variables: the integer number of Cooper pairs occupying the island and its conjugate, the $2 \pi$-cyclic superconducting phase difference between the island and the reservoir. The junction area must be chosen sufficiently small such that the electrostatic energy of the island due to an extra Cooper pair is larger than the Josephson energy of its coupling to the reservoir, thus confining fluctuations of the number of Cooper pairs below unity. Stated in electrical engineering language, one needs $Z_{J} \gtrsim R_{Q}$, where the junction reactive impedance $Z_{J}=\left(L_{J} / C_{J}\right)^{1 / 2}$ is defined by the Josephson characteristic inductance $L_{J}$ and capacitance $C_{J}(2)$, and where the superconducting impedance quantum is given by $R_{Q}=\hbar /(2 e)^{2} \approx 1 \mathrm{k} \Omega$, denoting Planck's constant $\hbar$ and the charge quantum $e$. The second type of circuit is based on a superconducting loop connecting the two electrodes of a small junction with an inductance which exceeds $L_{J}$. The circuit conjugate variables are now the magnetic flux generated by the persistent current in the loop and the displacement charge on the plates of the small junction capacitance. When $Z_{J} \gtrsim R_{Q}$, the large loop inductance is submitted to quantum fluctuations of flux larger than the flux quantum $\Phi_{0}=2 \pi \hbar / 2 e$, and therefore according to Heisenberg principle, the junction charge fluctuations are reduced below the value $2 e$.

In practice, the realization of both circuit types faces fundamental difficulties. Islands are exposed to random electric fields due to fluctuating charged impurities which are ubiquitous in most solid-state environments and whose compounded effect is described by a noisy offset 
charge. Although the fully developed charging effects were demonstrated for the Cooper pair box $(3,4)$, it soon became clear that the low-frequency offset charge noise was a major source of decoherence for charge qubits derived from this device (4-7). This state of affairs has prompted the development of alternative superconducting qubits based on large junctions with $Z_{J} \ll R_{Q}$, avoiding the single Cooper pair regime and the related charge offset problem $(8-10)$. On the other hand, implementing the island-free circuit, which is immune to charge offset noise, is another hard problem. This is because any finite-length wire with inductance $L$ always comes with self-capacitance $C$ which reduces the total charging energy of the circuit and therefore steers it away from the charging regime, unless $(L / C)^{1 / 2} \gg R_{Q}$. In fact, a purely electromagnetic inductance is incompatible with the single Cooper pair effects since $(L / C)^{1 / 2}$ is then bounded by the vacuum impedance $\left(\mu_{0} / \varepsilon_{0}\right)^{1 / 2} \approx 377 \Omega<R_{Q}, \mu_{0}$ and $\varepsilon_{0}$ being vacuum permeability and permittivity $(11,12)$.

In this paper, we present experimental results on a novel single Cooper pair circuit based on a superconducting loop, which solves both the inductance and the offset charge noise problems.

The small junction of our circuit is shunted by a series array of carefully chosen larger area tunnel junctions (Fig 1A-C). Here, all islands are connected to the rest of the circuit by at least one large junction so that quasistatic offset charges on all islands are screened. The large capacitances of the array junctions prevent phase slips within the array, and for excitations whose frequencies are below the junction plasma frequency, the array effectively behaves as an inductive wire. By choosing a sufficiently large number of array junctions it is possible to create an inductance exceeding that of the small junction. At low energies, the loop is effectively described by the loop flux $\hat{\Phi}$ and the small junction charge $\hat{Q}$, satisfying $[\hat{\Phi}, \hat{Q}]=i \hbar$.

To form a charge offset-free inductively shunted junction, four conditions involving the effective inductance $L_{J A}$ and capacitance $C_{J A}$ of the $\mathcal{N}$ array junctions are required: (i) $\mathcal{N} L_{J A} \gg$ $L_{J}$, (ii) $e^{-8 R_{Q} / Z_{J A}}<\varepsilon \ll 1$, (iii) $\mathcal{N} e^{-8 R_{Q} / Z_{J A}} \ll e^{-8 R_{Q} / Z_{J}}$, and (iv) $\mathcal{N}<\left(C_{J A} / C_{g}\right)^{1 / 2}$. In the first relation (i), we simply estimate the total array inductance to be $\mathcal{N} L_{J A}$ and require that it exceeds the small junction inductance, allowing it to support the large flux fluctuations of the loop. The second relation (ii), where $Z_{J A}=\left(L_{J A} / C_{J A}\right)^{1 / 2}$ is the array junction reactive impedance, dictates the minimum size of the array junctions necessary to reduce (13) the uncontrolled offset charge on the islands of the circuit below the desired value of the order of $2 e \times \varepsilon$. The third relation (iii) ensures that the inductive role of the array is not jeopardized by quantum phase slips (14). Specifically, the probability amplitude of a phase slip event within the array (1.h.s.) must be negligible compared to that in the small junction (r.h.s.). According to relation (iii) a fluxon tunnels in and out of the loop predominantly via the small junction, thus effectively erasing the discrete character of the array. Lastly, relation (iv) states that the inductance of the array is not shunted by the parasitic capacitances $C_{g}$ of array islands to ground. It is obtained by estimating the array parasitic resonance frequency to be $\left(L_{J A} \mathcal{N} \times C_{g} \mathcal{N}\right)^{-1 / 2}$, and requiring that it is larger than the junction plasma frequency $\left(L_{J A} C_{J A}\right)^{-1 / 2}$. Remarkably, it is the relation (iv) which, with present junction technology, most severely limits the maximum number of junction in the array and, thus, its maximum inductance.

We have implemented the above array proposal and constructed a new superconducting artificial atom which we have nicknamed "fluxonium". It contains $\mathcal{N}=43 \mathrm{Al}-\mathrm{AlOx}-\mathrm{Al}$ Josephson junctions (15) such that $Z_{J A} \simeq 0.5 R_{Q}$ and a small junction with $Z_{J} \simeq 1.5 R_{Q}$ (16). The above four conditions being realized, the fluxonium can be modelled (Fig. 1D) as a small junction shunted by an inductance $L_{A}$ (17). The three characteristic energies of this model, namely $E_{L}=\left(\Phi_{0} / 2 \pi\right)^{2} / L_{A}, E_{J}=\left(\Phi_{0} / 2 \pi\right)^{2} / L_{J}$ and $E_{C}=e^{2} /\left(2 C_{J}\right)$ have values corresponding to $0.52 \mathrm{GHz}, 9.0 \mathrm{GHz}$ and $2.5 \mathrm{GHz}$, respectively. The additional $L_{R} C_{R}$ resonator, capacitively 

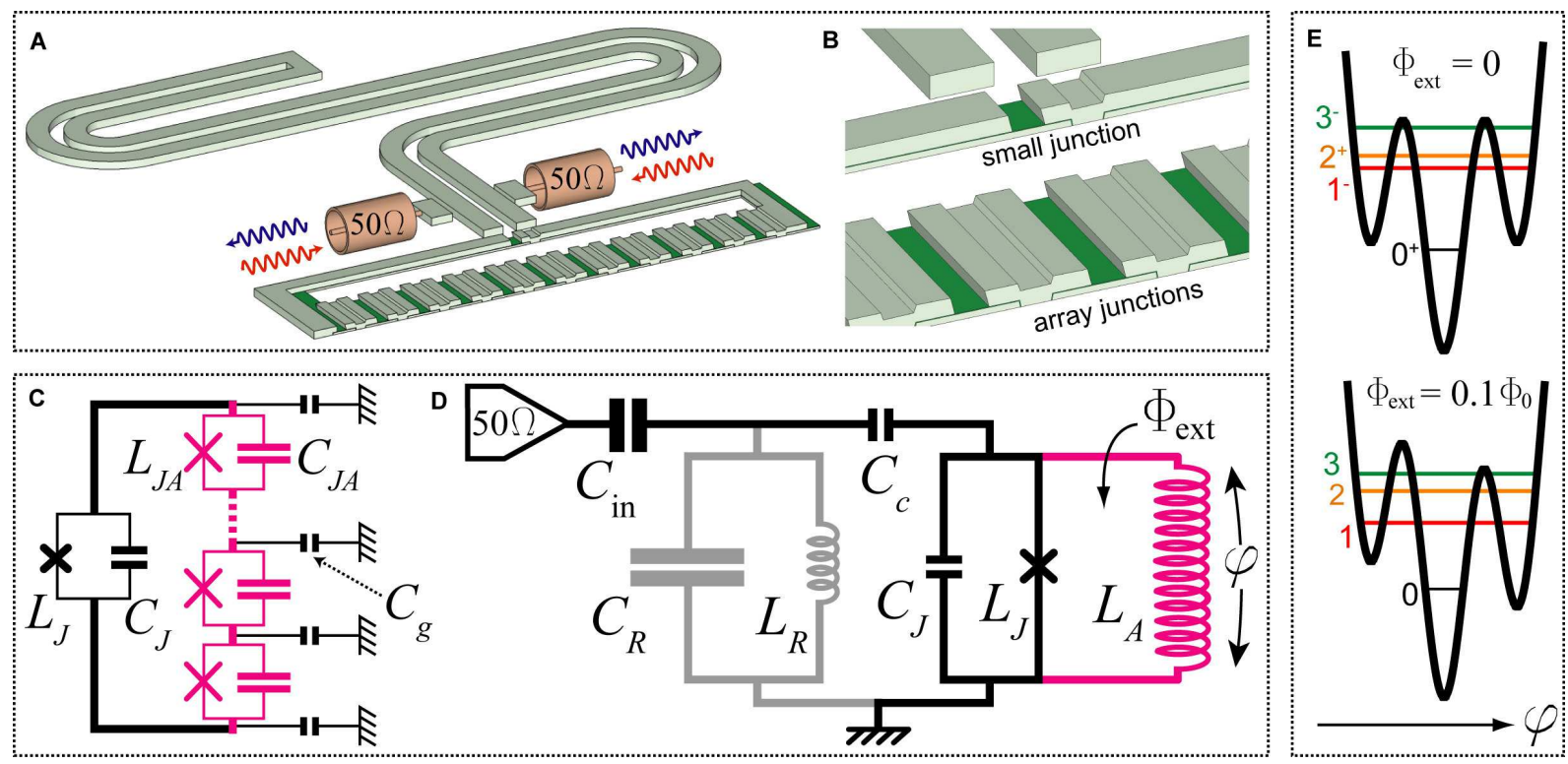

Figure 1: (A) Sketch of a small Josephson junction shunted by an array of larger area junctions. The two superconducting leads of the small junction are coupled capacitively to a quarter-wave microwave resonator, a "parallel wire" transmission line shorted on the opposite end. The resonator itself is probed capacitively and symmetrically via two $50 \Omega$ microwave ports, resulting in a loaded quality factor of 400 . The whole device is made with a single step standard Al/AlOx/Al double angle evaporation through an e-beam lithography mask on a high resistivity Si substrate. (B) Close-up view of the small junction region, showing top and bottom junction electrodes (grey) and their thin oxide layer (green). Array junctions are about one order of magnitude larger in area and spaced as tight as e-beam lithography resolution allows, minimizing microwave parasitics. (C) Electrical circuit representation of the loop formed by the small junction (black), with Josephson inductance $L_{J}$ and capacitance $C_{J}$, shunted by the array of larger junctions (purple), with the corresponding inductance $L_{J A}$ and capacitance $C_{J A}$. Islands formed between the array junctions have small capacitance to ground $C_{g}$. (D) Simplified circuit model of the fluxonium, consisting of three sections: i) circuit equivalent to a Cooper pair box, where the small junction with capacitance $C_{J}$ and non-linear Josephson inductance $L_{J}$ is capacitively, with capacitance $C_{c}$, coupled to the probe (solid black), such that $\left.L_{J} / C_{J}\right)^{1 / 2}>\hbar /(2 e)^{2}$; ii) giant inductance $L_{A} \gg L_{J}$ provided by the junction array (purple); iii) a parallel combination of $C_{R}$ and $L_{R}$ such that $\left(L_{R} / C_{R}\right)^{1 / 2} \approx 50 \Omega \ll \hbar /(2 e)^{2}$ which is the circuit model for distributed transmission line resonator (grey). (E) Potential seen by the reduced flux $\varphi$ and energy spectrum of the circuit (D) for two values of external flux $\Phi_{\text {ext }}$. At $\Phi_{\text {ext }}=0$, energy levels possess well-defined parity as indicated with ' + ' and '-' signs next to level numbers. Note that, in contrast with the RF-SQUID or flux qubit, there is on average only one level per local minima. 


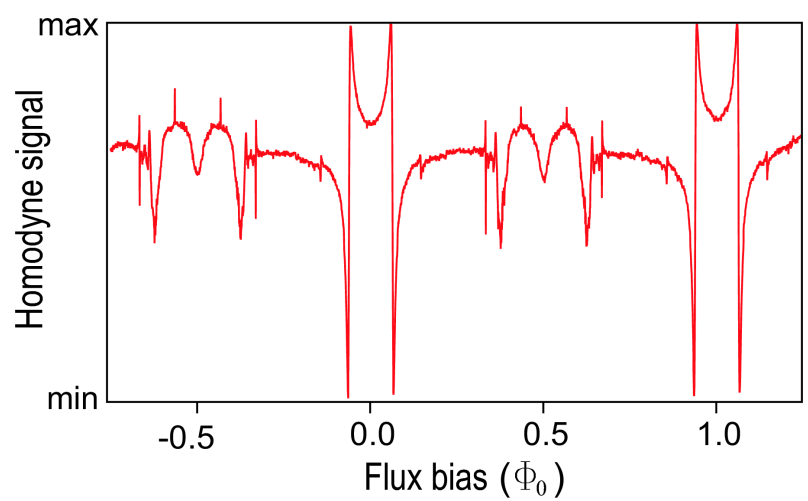

Figure 2: Modulation of the reflected $8.18 \mathrm{GHz}$ microwave signal with externally applied flux $\Phi_{\text {ext }}$. The signal is clearly flux-periodic indicating that the junction ring is closed and superconducting. The values of $\Phi_{\text {ext }}$ at which the signal undergoes full swings correspond to the anticrossings of the $0-1$ transition frequency of the device with the resonator bare frequency, later inferred to be $8.1755 \mathrm{GHz}$. The measurement tone populates the resonator with less than 0.01 photon on average.

connected to the small junction (Fig. 1D), reads out the "atom" in a manner analogous to the dispersive measurement of cQED qubits (18). It is implemented by a quarter-wave superconducting coupled microstrip resonator (Fig. 1A) with quality factor 400 due to capacitive coupling to the two $50 \Omega$ measurement ports. The resonator frequency $\omega_{R}=\left(L_{R} C_{R}\right)^{-1 / 2} \simeq 2 \pi \times 8.17 \mathrm{GHz}$ is pulled by the reactance of the fluxonium circuit and is monitored by standard ultra low noise microwave reflection technique. The fluxonium reactance depends on its quantum state, an effect leading to a purely dispersive state measurement (15). An externally imposed, static magnetic flux $\Phi_{\text {ext }}$ threading the loop $\Phi_{0}$-periodically modulates the spacings of energy levels of our artificial atom.

Introducing the operators $\hat{N}=\hat{Q} / 2 e$ and $\hat{\varphi}=2 e \hat{\Phi} / \hbar$, describing the reduced charge on the junction capacitance and its conjugate reduced flux operator (1), the Hamiltonian of the fluxonium coupled to its readout resonator can be written as

$$
\hat{H}=4 E_{C} \hat{N}^{2}+\frac{1}{2} E_{L} \hat{\varphi}^{2}-E_{J} \cos \left(\hat{\varphi}-2 \pi \Phi_{\text {ext }} / \Phi_{0}\right)+g \hat{N}\left(\hat{a}+\hat{a}^{\dagger}\right)+\hbar \omega_{R} \hat{a}^{\dagger} \hat{a}
$$

Here $\hat{a}$ is the photon annihilation operator for the resonator, $g$ is the atom-resonator coupling constant. The second term and the range of definition of $\hat{\varphi}$ and $\hat{N}$, whose eigenvalues are here both on the entire real axis, distinguishes the form of Hamiltonian (1) from that of the Cooper pair box in cQED experiments (18). There are three important points to note concerning this Hamiltonian (2): i) it is invariant under the transformation $\hat{N} \rightarrow \hat{N}+N_{\text {offset }}$ ( $N_{\text {offset }}$ stands for offset charge value) hence the "charge-free" character of our device; ii) it differs from that of the transmon (13) since offset charge influence is screened for all states, not just for the low-lying states; iii) its second term, despite the fact that $E_{L}$ is the smallest of the fluxonium energies, has a non-perturbative influence on the full energy spectrum of this artificial atom, which presents strongly anharmonic transitions (21) (Fig. 1E). Our experiment probes these transitions by microwave spectroscopy, from which we infer the size of charge fluctuations.

To characterize the fluxonium, we first measure the ground state resonator pull as a function of $\Phi_{\text {ext }}$. The results (Fig. 2) show the expected $\Phi_{0}$ - periodicity as well as the avoided crossings of the resonator frequency and the ground to excited state transitions. This confirms that the 


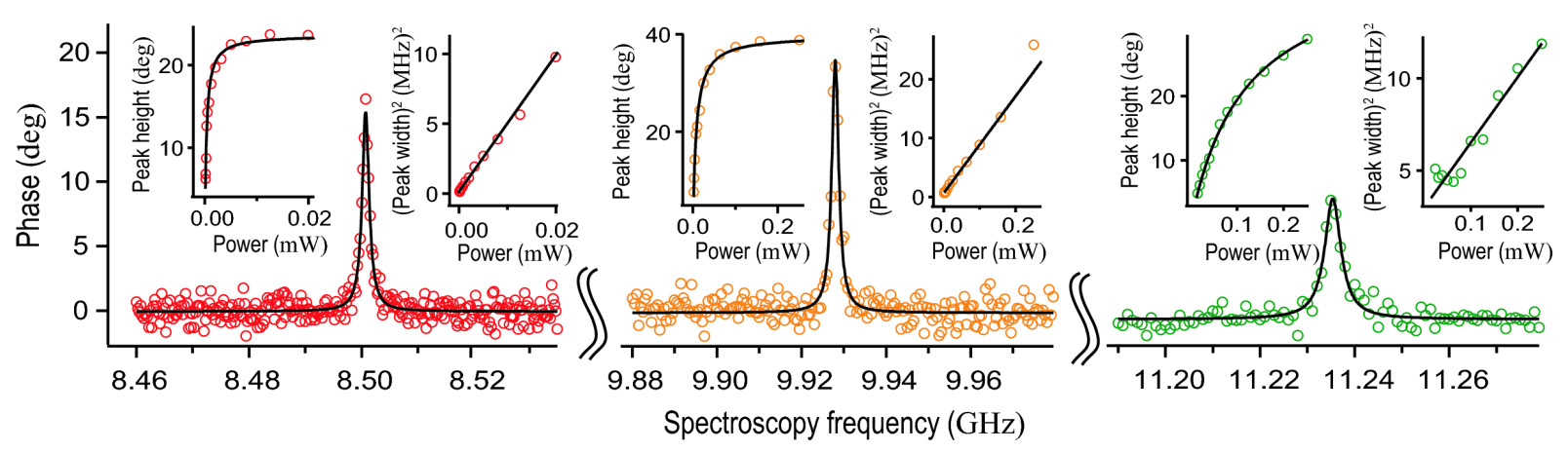

Figure 3: Phase (colored circles) of reflected readout tone as a function of spectroscopy tone frequency taken at $\Phi_{\text {ext }}=0.05 \Phi_{0}$. Data for the first three resonances (further identified as transitions from the ground state to states 1,2 and 3) is shown from left to right in red, orange and green respectively. Resonances are well-fitted by Lorentzians (solid black lines) for a broad range of spectroscopy powers. Insets on the two sides of each resonance show the dependence of the resonant peak height (left) and width squared (right) on the spectroscopy tone power. Data in all insets follow the predictions (solid black lines) of Bloch equations describing relaxation dynamics for a spin $1 / 2$ and indicate that all transitions involve one photon.

entire 44 junction loop is superconducting and that the resonator-atom system is in the strong coupling regime of cavity QED (22).

Next, we perform a two-tone spectroscopy measurement (23) at a fixed flux $\Phi_{\text {ext }}=0.05 \Phi_{0}$, during which, in addition to the fixed frequency readout tone, we probe the transition frequencies of the atom through a second, variable frequency spectroscopy tone. The resulting peaks (Fig. 3), correspond to the later-determined $0-1,0-2$, and $0-3$ transitions from the atom ground state. The peaks are well-fitted by Lorentzians and their power-dependent widths and heights are well-explained by the Bloch equations of precessing spin 1/2 (24) as shown in the insets of (Fig. 3). Extrapolating fitted linewidths to zero spectroscopy power, we obtain lower bound estimates of their decoherence time at 350, 250 and 80 ns respectively.

Our main result is the spectroscopic data collected as a function of both spectroscopy frequency and flux (Fig. 4A). Note that $\Phi_{\text {ext }}$ variations span $20 \%$ of $\Phi_{0}$ around $\Phi_{\text {ext }}=0$ instead of the usual $1 \%$ or less around $\Phi_{0} / 2$ in flux qubit experiments (9). In (Fig. 4B) we compare the measured peak center frequencies with the prediction for the $0-1,0-2,0-3$ and the twophoton $0-4$ transitions obtained from numerical diagonalization of Hamiltonian (Eq. 2). Note that we are in effect fitting more than three flux dependent functions, i.e. the flux dependent transition frequencies, with only three a priori unknown energies $E_{C}, E_{L}$ and $E_{J}$ so the problem is severely overconstrained. The fit of the line (Fig. 4B) labeled SR (for array self-resonance) requires a minor extension of the model taking into account parasitic capacitances across the array (15). Apart from introducing another resonator mode coupled to the atom, this extension by no means invalidates the inductive character of the array, at least as far as the $0-1$ and $0-2$ transition of the fluxonium are concerned. Even the perturbation of the $0-3$ and $0-4$ transition frequencies by this extra mode is less than $2 \%$.

Based on the excellent agreement between theory and experiment, we infer the wavefunctions of the first three energy levels, and plot their amplitudes both in charge (Fig. 4C) and flux (Fig. 4D) representations for $\Phi_{\text {ext }}=0$. In the ground state, we find that the ratio of charge to flux fluctuations is $\Delta N / \Delta \varphi=0.56$, about 5 times smaller than the fine structure constant allows for a conventional resonator. This confirms that the charge in our circuit is indeed lo- 

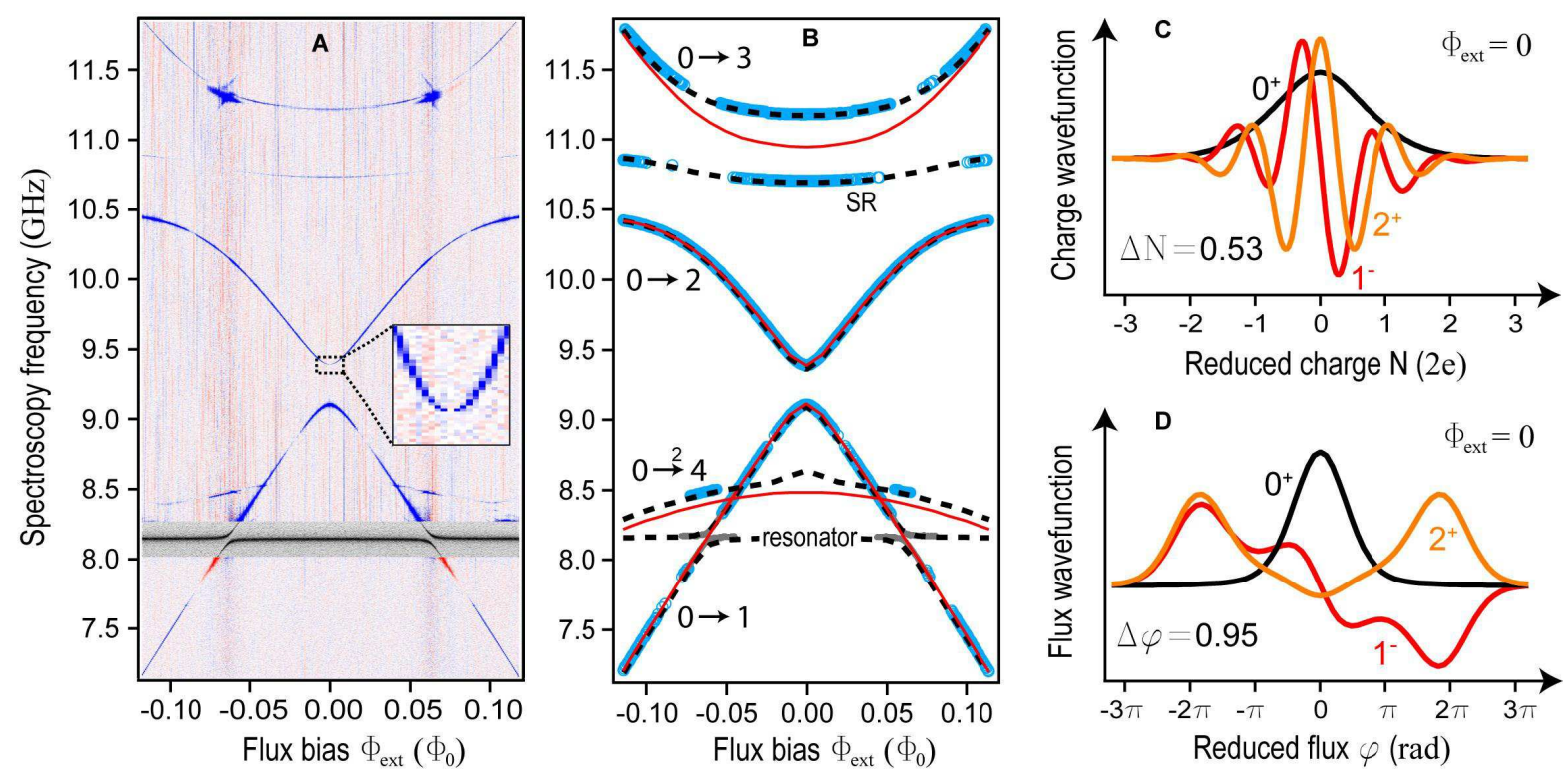

Figure 4: (A) Phase of reflected readout tone as a function of the spectroscopy tone frequency and external flux. The color scale encodes the value of phase, zero corresponding to the mauve background, blue to positive values (peaks) and red to negative values (dips). The grey region shows the reflected phase of a single tone, swept close to the resonator bare frequency exhibiting a $50 \mathrm{MHz}$ vacuum Rabi splitting of the resonator with the fluxonium transition $0-1$. The inset in (A) zooms in the central region of the $0-2$ transition and confirms that it is indeed symmetry-forbidden at $\Phi_{\text {ext }}=0$. (B) Measured peak frequencies (blue circles) fitted by numerically computed spectrum of Hamiltonian (2) (solid red lines) and its modification (see supplementary online text) to explain the additional transition labeled "SR" (dashed black lines). (C) Amplitude of fluxonium wave functions for levels 0 (black), 1 (red) and 2 (orange) computed in charge representation at zero flux bias, using circuit parameters extracted from the fits. (D) Same as in (C) but in flux representation. The flux representation wave functions demonstrate that the reduced flux is delocalized compared to the size of the Josephson well while charge wave functions confirm that the localization of charge on the junction is less than a single Cooper pair charge. Note that in this circuit, the junction charge is a continuous variable, in contrast to the Cooper pair box, and flux swings of more than $2 \pi$ are allowed. 
calized at the single Cooper pair level $(\Delta N=0.53, \Delta \varphi=0.95)$. The wavefunctions in flux representation (Fig. 4D) can be interpreted as simple superpositions of states in which the reduced flux $\varphi$ is localized in the wells of the Josephson cosine potential (fluxon states, hence the name fluxonium). The parity of fluxonium states, which forbids the $0-2$ transition at zero external flux, manifests itself explicitly by a remarkable "hole" in the corresponding spectroscopic line (Fig. 4A, inset). The allowed transition between the second and third level (data not shown) is particularly spectacular since it corresponds to motion of the total flux in the fluxonium loop by two whole flux quanta. This is to be contrasted with the $10 \%$ of flux quantum or less flux motion involved in transitions of the flux and phase qubits $(8,9)$. Nevertheless, despite the large flux fluctuations of the system and the corresponding charge pinning, the circuit has complete immunity to offset charge variations: the data of (Fig. 4A) has been taken piecemeal in 72 hours and no jumps or drifts have been observed during this period.

We have thus demonstrated that an array of Josephson junctions with appropriately chosen parameters can perform two functions simultaneously: short-circuit the offset charge variations of a small junction and protect the strong non-linearity of its Josephson inductance from quantum fluctuations. The data shows that the array possesses a microwave inductance $10^{4}$ times larger than the geometric inductance of a wire of the same $20 \mu \mathrm{m}$ length. The reactance of such inductor is about $3 R_{Q} \approx 20 \mathrm{k} \Omega$ at $10 \mathrm{GHz}$ while its resistance is less than $1 \Omega$. The spectrum of the fluxonium qubit suggests it is as anharmonic as the flux qubit but as insensitive to flux variations as the transmon qubit. Possible applications of this single Cooper pair charging effect immune to charge noise include the observation of fully developed macroscopic quantum coherent oscillations between fluxon states (25), the search for a " $\Lambda$ " or " $V$ " transition configurations for the shelving of quantum information (26) in superconducting artificial atoms, topological protection of superconducting qubits (27), and finally the long-sought quantum metrology of electrical current via Bloch oscillations $(28,29)$.

\section{References and Notes}

1. H. Grabert, M. H. Devoret (Eds.), Single Charge Tunneling: Coulomb Blockade Phenomena in Nanostructures

2. B. D. Josephson, Rev. Mod. Phys 36, 216 (1964).

3. V. Bouchiat, D. Vion, P. Joyez, D. Esteve, M. H. Devoret, Physica Scripta T76, 165-170 (1998).

4. Y. Nakamura, Yu. A. Pashkin, and J. S. Tsai, Nature 398, 786 (1999).

5. D. Vion et al., Science 296, 866 (2002).

6. K. Bladh, T. Duty, D. Gunnarsson P. Delsing, New J. Phys. 7, 180 (2005).

7. M. Metcalfe et al., Phys. Rev. B 76, 174516 (2007).

8. J. M. Martinis, S. Nam, J. Aumentado, C. Urbina, Phys. Rev. Lett. 89, 117901 (2002).

9. I. Chiorescu, Y. Nakamura, C. J. P. M. Harmans, J. E. Mooij, Science 299, 1869 (2003).

10. J. A. Schreier et al., Phys. Rev. B 77, 180502(R) (2008). 
11. R. P. Feynman, R. B. Leighton, M. Sands, The Feynman Lectures on Physics (AddisonWesley, 1964), vol. 2, chap. 23 (Springer, 1992).

12. Note that the ratio $\left(\mu_{0} / \varepsilon_{0}\right)^{1 / 2} / R_{Q}=16 \pi \alpha$, where $\alpha \approx 1 / 137$ is the fine structure constant.

13. J. Koch et al., Phys. Rev. A 76, 042319 (2007).

14. K. A. Matveev, A. I. Larkin, L. I. Glazman, Phys. Rev. Lett. 89, 096802 (2002).

15. Materials and methods are available as supporting material on Science online.

16. Due to coupling of the fluxonium circuit to the readout resonator (Fig.1D) $C_{J}$ is renormalized to the value $C_{J}+C_{c}$, with corrections of order $C_{c} / C_{R} \simeq 1 \%$.

17. Here we acknowledge experiments $(30,31)$ in which a small Josephson junction was DCbiased in series with a micron-scale disordered film resistance exceeding $R_{K}(30)$, or with an array of small-junction SQUIDs (31) with zero-bias resistance tuned by magnetic field to exceed $R_{K}$. Both experiments aimed at protecting the small junction from the shunting effect of low impedance environment using highly dissipative biasing elements. While results of DC I-V measurements were consistent with single Cooper pair effects, they were distorted by Joule heating and other out-of-equilibrium effects in these biasing elements. We avoid problem of dissipation with an array employing the pure Josephson kinetic inductance.

18. A. Wallraff et al., Nature 431, 162 (2004).

19. M. Devoret in Quantum Fluctuations in Electrical Circuits, S. Reynaud, E. Giacobiano, J. Zinn-Justin, eds. (Elsevier Science, 1997).

20. J. Koch, V. Manucharyan, L. Glazman, M. Devoret, arXiv:0902.2980 (2009).

21. Note that while the circuit in (Fig. 1D) might appear identical to the RF-SQUID circuit (32), there is a drastic difference between the two when charging, Josephson and inductive energies are compared. In addition, RF-SQUID is addressed by coupling to its flux degree of freedom via mutual inductance, while the fluxonium is addressed by coupling to its charge degree of freedom via capacitance $C_{c}$.

22. J. Raimond, M. Brune, S. Haroche, Rev. Mod. Phys. 73, 565 (2001).

23. D. Schuster et al., Phys. Rev. Lett. 94, 123602 (2005).

24. A. Abragam, The principles of nuclear magnetism (Oxford University Press, 1961).

25. A. J. Leggett, J. Phys.: Condens. Matter 14, R415 (2002) and references therein.

26. W. Nagourney, J. Sandberg, H. Dehmelt, Phys. Rev. Lett. 56, 2797 (1986).

27. A. Kitaev, arXiv:cond-mat/0609441 (2006).

28. D. V. Averin, A. B. Zorin, K. K. Likharev, Zh. Exp. Teor. Phys. 88, 692 (1985).

29. K. K. Likharev, A. B. Zorin, J. Low Temp. Phys. 59, 347 (1985). 
30. L. S. Kuzmin, D. B. Haviland, Phys. Rev. Lett. 67, 2890 (1991).

31. M. Watanabe, D. B. Haviland, Phys. Rev. Lett. 86, 5120 (2001).

32. J. R. Friedman, V. Patel, W. Chen, S. K. Tolpygo, J. E. Lukens, Nature 406, 43 (2000).

33. We acknowledge discussions with Markus Brink, Etienne Boaknin, Michael Metcalfe, R. Vijay, David Schuster, Leo DiCarlo, Luigi Frunzio, Robert Schoelkopf and Steven Girvin. This research was supported by the NSF under grants DMR-0754613, DMR-032-5580, the NSA through ARO Grant No. W911NF-05-01-0365, the Keck foundation, and Agence Nationale pour la Recherche under grant ANR07-CEXC-003. M.H.D. acknowledges partial support from College de France. 


\section{Supporting Material}

\section{Materials and Methods}

Sample fabrication. The device is made on a high-resistivity Si substrate, $300 \mu \mathrm{m}$ thick. Both Josephson junctions and the readout resonator are fabricated in a single step using e-beam lithography, double angle $\mathrm{Al}$ e-beam evaporation and lift-off techniques. The Al evaporation and oxidation is conducted in an e-gun evaporator at pressures less than $10^{-5} \mathrm{~Pa}$, AlOx grown in the environment of $680 \mathrm{~Pa}$ of $15 \%$ oxigen-in-argon mixture during 10 minutes. The areas of the small junction and array junctions are designed to be $0.2 \times 0.3 \mu \mathrm{m}^{2}$ and $0.25 \times 2 \mu \mathrm{m}^{2}$, respectively. All 43 array junctions are equally spaced at less than $200 \mathrm{~nm}$ so that total length of the array is only $20 \mu \mathrm{m}$. The loop area of the array-small junction ring is $3 \times 20 \mu \mathrm{m}^{2}$.

Sample mount. The Si chip is glued using GE varnish to the copper base of a fully enclosing, custom-made microwave sample holder, shielding the sample from both residual RF, infrared and optical photons. The holder provides two well-matched transitions from the Anritsu Kconnectors on the outside of the holder to the two microstrip lines made on a PCB inside the holder. The resonator's on-chip launching pads, schematically indicated as two sections of a coaxial cable and marked " $50 \Omega$ " in (Fig. 1A), are then wirebonded to the ends of the two microstip lines.

Cryogenic setup. The experiment is performed in a dilution refrigerator with base temperature $10-20 \mathrm{mK}$. Both resonator and the qubit are differentially excited via the $\Delta$-port of a $180^{\circ}$ hybrid (Krytar, $2-18 \mathrm{GHz}$ ), whose two outputs are connected to the two ports of the sample holder. Incoming and outgoing signals are separated with a directional coupler (Krytar, $2-20 \mathrm{GHz}$ ). The incoming signal line is attenuated using 10 and $20 \mathrm{~dB}$ microwave attenuators (XMA) at all temperature stages of the refrigerator, to remove non-equilibrium noise. The output line is amplified at the $4 \mathrm{~K}$ stage with a low-noise HEMT amplifier (Caltech, $1-12 \mathrm{GHz}$, $30 \mathrm{~dB}$ gain). Two cryogenic isolators (Pamtech, $4-12 \mathrm{GHz}, 15 \mathrm{~dB}$ ) are placed between the amplifier and the sample, at the $800 \mathrm{mK}$ stage and at the base stage, again to remove nonequilibrium noise, especially that coming from the amplifier. Stainless steel SMA cables are used to connect between the different temperature stages. All components are thermally anchored to the proper refrigerator stages. A $\sim 1 \mathrm{~cm}$ diameter custom made superconducting coil is glued to the sample holder, a few $\mathrm{mm}$ away from the chip, to provide perpendicular magnetic flux bias. The sample holder together with the coil is placed into a Cryoperm cylinder to shield it from stray quasistatic magnetic fields.

Room temperature measurement setup. The readout resonator is excited with Agilent E8257D signal generator, the spectroscopy signal is generated using Agilent E8267D vector signal generator and Tektronix 520 AWG. Both signals are combined at room temperature and sent into the input line of the refrigerator. The reflected $\sim 8 \mathrm{GHz}$ readout signal from the refrigerator output line is amplified at room temperature with two Miteq amplifiers ( $1-12 \mathrm{GHz}, 30 \mathrm{~dB}$ gain), mixed down with a local oscillator (a third Agilent E8257D) to an IF signal of $0-50 \mathrm{MHz}$, filtered and amplified with the IF amplifier (SRS SR445A), and finally digitized using $1 \mathrm{GS} / \mathrm{s}$ Agilent Acqiris digitizer. A software procedure then extracts the phase and the amplitude of the digitized wave. The experiment is typically repeated $10^{4}$ times to average the Gaussian noise to an acceptable level. Because the duration of each experiment is about 10 microseconds, every averaged data point is taken in a fraction of a second. All microwave test equipment is phase locked using a $\mathrm{Rb}$ precision $10 \mathrm{MHz}$ reference (SRS FS725). The magnetic coil is biased in 

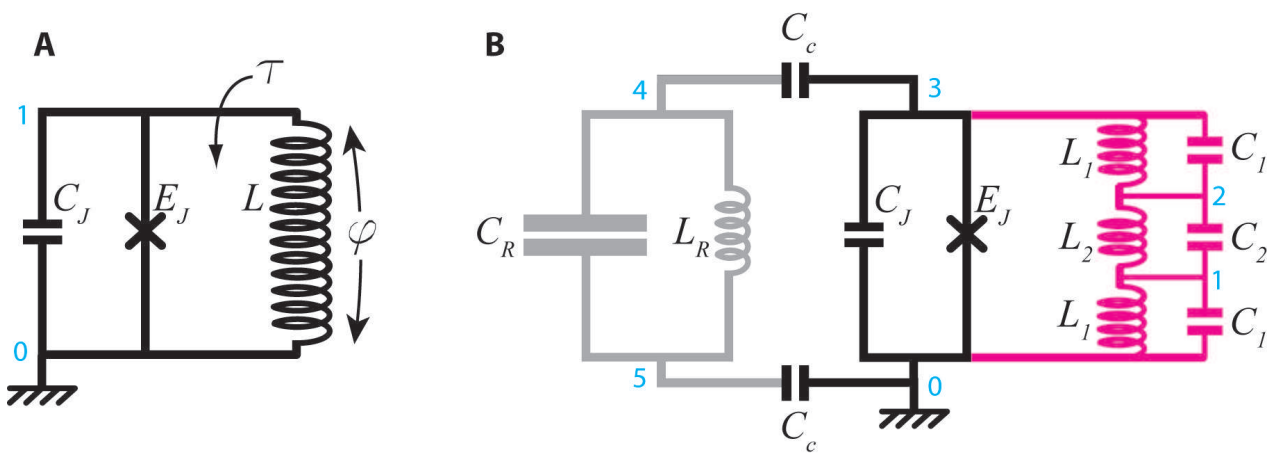

Figure 5: Models for the fluxionium device. (A) Inductively shunted junction model. (B) Extended fluxionium model, including the capacitive coupling to the mode of a transmissionline resonator, and parasitic capacitances across the array. Numbers in cyan enumerate the nodes of the circuits.

series with a resistor with Yokogawa 7751 voltage source.

Comments on the data. The data in (Fig. 2) shows the digitized homodyne (zero IF) signal as a function of magnetic field, with the spectroscopy generator turned off. The data in (Fig. 3 ) shows the phase of the digitized heterodyne (50 MHz IF) signal, as a function of frequency of the spectroscopy generator. The data in (Fig. 4A) is taken in the pulsed regime, when the spectroscopy generator outputs a $6 \mu$ s saturating pulse followed immediately by the $2 \mu$ s readout pulse. This way we ensure that the sample is exposed to only one tone at a time, avoiding various spurious effects. The image presented in (Fig. 4A) contains $367 \times 4597$ data points.

\section{Supplementary Text}

In our analysis of the fluxonium device, we use two simple models whose corresponding circuits are depicted in Figure 5: (A) the inductively shunted junction model, (B) the extended fluxonium model, describing the fluxonium coupled to a transmission-line resonator.

Inductively shunted junction model. The simplest model of the fluxonium device is the inductively shunted junction model, see Fig. 5(A). It neglects parasitic capacitances across the fluxonium's Josephson junction array, and assumes that all internal degrees of freedom of the array are frozen out. In this limit, the fluxonium consists of a single Josephson junction with capacitance $C_{J}$ and Josephson energy $E_{J}$, shunted by a large inductance $L$. Quantization of this circuit ( 1 ) is straightforward and leads to the Hamiltonian

$$
\hat{H}_{0}=4 E_{C} \hat{N}^{2}+\frac{1}{2} E_{L} \hat{\varphi}^{2}-E_{J} \cos \left(\hat{\varphi}-2 \pi \frac{\Phi_{\text {ext }}}{\Phi_{0}}\right),
$$

where the charge on the junction capacitance $\hat{N}$ (in units of $2 e$ ) and the reduced flux $\hat{\varphi}$ are canonically conjugate variables, $[\hat{\varphi}, \hat{N}]=i$. Structurally, this Hamiltonian is identical to the Hamiltonian describing one-junction flux qubits, and flux-biased phase qubits. However, the regime of large inductances relevant for the fluxionium differs from typical parameters in flux and phase qubits, and has been discussed in Ref. (2).

Extended fluxonium model. For a more complete modelling of the spectra obtained in the experiment, we take into account the coupling of the fluxonium device to a transmission-line resonator. In addition to this resonant mode, the experimental data shows another resonance 
coupling to the fluxonium device. Such additional resonances are expected when accounting for the parasitic capacitances of the Josephson junction array. The simplest effective model accurately describing the experimental data includes very few of these capacitances, and approximates the array by a combination of inductances and capacitances as shown in Fig. 5(B).

The Lagrangian describing this circuit can be written in the form

$$
\begin{aligned}
\mathcal{L}=\frac{C_{J}^{\prime}}{2} \dot{\phi}_{3}-\frac{1}{2 L}\left(\phi_{3}-\Phi_{\text {ext }}\right)^{2}+ & E_{J} \cos \left(\frac{2 \pi \phi_{3}}{\Phi_{0}}\right)+C^{\prime} \dot{\theta}_{2}-\frac{1}{L^{\prime}} \theta_{2}^{2}+\frac{C_{R}^{\prime}}{2} \dot{\varphi}_{4}^{2}-\frac{1}{2 L_{R}} \varphi_{4}^{2} \\
& +\frac{C_{c}}{2} \dot{\phi}_{3} \dot{\varphi}_{4}+\tilde{C} \dot{\theta}_{2} \dot{\phi}_{3},
\end{aligned}
$$

where $C_{J}^{\prime}=C_{c} / 2+C_{J}+2 C_{1} \lambda_{1}^{2}+C_{2} \lambda_{2}^{2}, \lambda_{i}=L_{i} / L, C^{\prime}=9\left(C_{1}+2 C_{2}\right) \lambda_{1}^{2} \lambda_{2}^{2}, L^{\prime}=L /\left(9 \lambda_{1} \lambda_{2}\right)$, $C_{R}^{\prime}=C_{R}+C_{c} / 2, \tilde{C}=6 \lambda_{1} \lambda_{2}\left(C_{1} \lambda_{1}-C_{2} \lambda_{2}\right)$, and we have disposed of another resonant mode which does not couple to the fluxonium device. In terms of the original generalized flux $\phi_{i}$ at each node $i$, the relevant variables are $\phi_{3}$ (associated with the fluxonium subsystem), the resonator mode $\varphi_{4}=\phi_{4}-\phi_{5}$, and the additional resonant mode $\theta_{2}=-\frac{1}{6 \lambda_{1} \lambda_{2}}\left(\phi_{2}-\phi_{1}-\lambda_{2} \phi_{3}\right)$. Employing canonical quantization of this circuit, we find the effective Hamiltonian

$$
\hat{H}=\hat{H}_{0}+\sum_{j=1,2} \hbar \omega_{j} \hat{a}_{j}^{\dagger} \hat{a}_{j}+\hbar \sum_{j=1,2} g_{j} \hat{N}\left(\hat{a}_{j}^{\dagger}+\hat{a}_{j}\right)
$$

describing the inductively shunted junction, $\hat{H}_{0}$, coupled to two resonant modes $j=1,2$ with coupling strengths $g_{1}$ and $g_{2}$, respectively.

Theory fits to experimental data. Design and fabrication of the fluxonium system only allow for imprecise estimates of the system parameters. Thus, the comparison between experimental data and theoretical prediction requires the fitting of theory curves to determine the system parameters with more accuracy. The parameters at our disposal are: $E_{J}, E_{C}$, and $E_{L}$ (for both the inductively shunted junction and the extended fluxonium models). In addition, the extended fluxonium model takes the resonant mode frequencies and coupling strengths $\omega_{1,2}$ and $g_{1,2}$ as input. Fits are obtained by extracting the center frequencies from the experimental data and employing a least-squares fit algorithm.

Fit to inductively shunted junction model. A simultaneous fit to the full flux-dependence of the $0-1$ and 0-2 transitions around the zero-flux point fully determines the fluxonium parameters $E_{C}, E_{J}$, and $E_{L}$ (see Table 1 for the obtained parameter values). A comparison between the resulting theory prediction of higher transitions can then be used as a consistency check. While the agreement for the 0-1 and 0-2 transitions is good, we find systematic deviations for higher levels. The reason for these deviations lies in the effect of the additional resonance on the 0-2 transition: the additional resonance leads to significant frequency shifts of the 0-2 transitions. Ignoring this effect leads to a systematic error in the estimation of the fluxonium parameters.

Fit to extended fluxonium model. For best agreement, both resonator and additional resonant mode are taken into account. Using the full experimental data we obtain a fit for the extended fluxonium model, which shows excellent agreement with the data. The resulting parameter values are given in Table 1 .

\section{References and Notes}

1. M. Devoret in Quantum Fluctuations in Electrical Circuits, S. Reynaud, E. Giacobiano, J. Zinn-Justin, eds. (Elsevier Science, 1997).

2. J. Koch, V. Manucharyan, L. Glazman, M. Devoret, arXiv:0902.2980 (2009). 


\begin{tabular}{lcc}
\hline \hline & $\begin{array}{c}\text { inductively shunted } \\
\text { junction model }\end{array}$ & extended fluxonium model \\
\hline$E_{C} / h$ & 2.39 & 2.47 \\
$E_{J} / h$ & 8.93 & 8.97 \\
$E_{L} / h$ & 0.52 & 0.52 \\
$\omega_{1} / 2 \pi$ & n.a. & 8.18 \\
$\omega_{2} / 2 \pi$ & n.a. & 10.78 \\
$g_{1} / 2 \pi$ & n.a. & 0.135 \\
$g_{2} / 2 \pi$ & n.a. & 0.324 \\
\hline \hline
\end{tabular}

Table 1: Fluxonium system parameters obtained from least-squares fits to the inductively shunted junction and the extended fluxonium model. All values are given in GHz. The coupling constants are expressed in terms of the coupling strength for the fluxonium 0-1 transition. 